\title{
Symmetries and Conservation Laws of a Spectral Nonlinear Model for Atmospheric Baroclinic Jets
}

\author{
N.H. Ibragimov ${ }^{1,2}$, R.N. Ibragimov ${ }^{3,4 *}$, L.R. Galiakberova ${ }^{1}$ \\ ${ }^{1}$ Laboratory "Group Analysis of Mathematical Models in Natural and Engineering Sciences", Ufa \\ State Aviation Technical University, 450000 Ufa, Russia \\ ${ }^{2}$ Center for Mathematical Modeling with Lie Group Analysis (CeMMLiGA) \\ Blekinge Institute of Technology, SE-371 79 Karlskrona, Sweden \\ ${ }^{3}$ Department of Mathematics, University of Texas at Brownsville \\ Brownsville, TX 78520, USA \\ ${ }^{4}$ Pacific Northwest National Laboratory, Richland, WA 99352, USA
}

\begin{abstract}
In this paper, we shall obtain the symmetries of the mathematical model describing spontaneous relaxation of eastward jets into a meandering state and use these symmetries for constructing the conservation laws. The basic eastward jet is a spectral parameter of the model, which is in geostrophic equilibrium with the basic density structure and which guarantees the existence of nontrivial conservation laws.
\end{abstract}

Keywords and phrases: 76U05, 35Q30, 35Q35, 35Q86

Mathematics Subject Classification: atmospheric jets, effects of rotation, conservation laws

\section{Introduction}

The weather maps at mid-latitudes invariably show the presence of wave-like horizontal excursions of temperature and pressure contours, superposed on eastward mean flows such as jet stream ([1],[2], [3]). For illustration purposes, Figure $1^{1}$ is used to show the photo of the cold front is marked by a blue line, Sandy by the red symbol, the subtropical jet stream by a green arrow (bottom center), and the blocking pattern by the "L" in the upper right. The subtropical jet stream is already deforming Sandy from the symmetrical shape expected of a classic hurricane into the "comma" shape typical of Northeasters. The trough in the polar jet stream lies behind (west) of the cold front. The blocking pattern keeps Sandy from escaping to the east. Although not obvious on surface level maps, or even on the 850 mb level map below, Sandy is sandwiched between the polar and subtropical jet streams.

Similar undulations are also found in the ocean on eastward currents such as the Gulf Stream in the north Atlantic ([4], [5], [6]). A typical wavelength of these disturbances is observed to be of the order of the internal Rossby radius, that is about $4000 \mathrm{~km}$ in the atmosphere and $100 \mathrm{~km}$ in the ocean. They

\footnotetext{
* Corresponding author. E-mail: ibrranis@gmail.com

${ }^{1}$ Source: http://www.geologyinmotion.com/2012/11/hurricane-sandy-and-baroclinic.html
} 


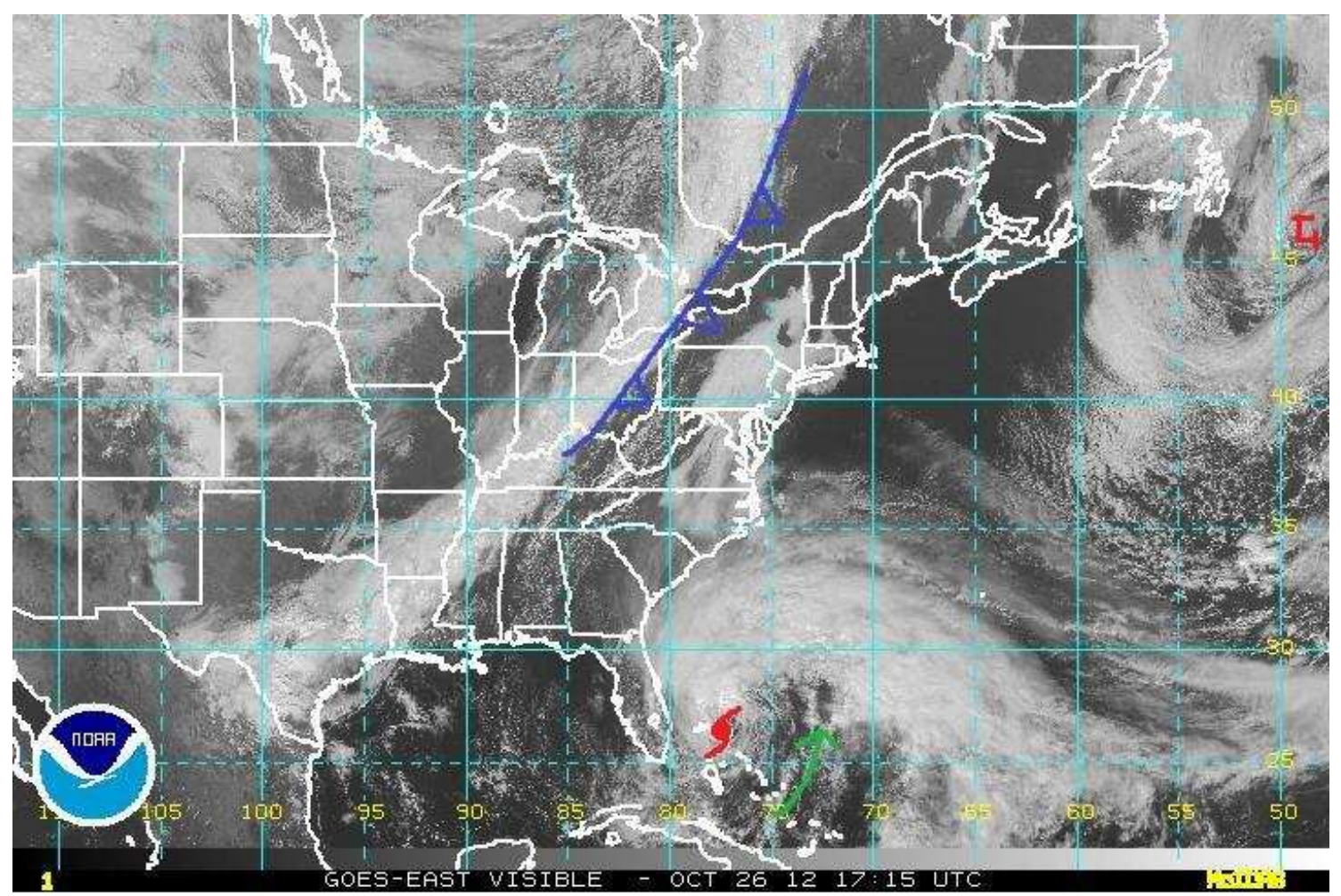

FiguRE 1. Hurricane Sandy (right side) and the line of clouds marking the cold air front coming in from Canada NOAA image taken from Joe Zagrodnik's.

seem to be propagating as Rossby waves, but their erratic and unexpected appearance suggests that they are not forced by any external agency, but are due to inherent instability of mid-latitude eastward flows ([7], [8], [9]). This means that the eastward flows have a spontaneous tendency to develop wave-like disturbances ([10], [11]).

The poleward decrease of the solar irradiation results in a poleward decrease of the temperature and a consequent increase of the density [12]. One possible idealized distribution of the atmospheric density in the northern hemisphere corresponds to the case when the density increases northward due to the lower temperatures near the poles and decreases upward because of static stability [15]. According to the thermal wind relation, an eastward flow (such as the Jet Steam in the atmosphere or the Gulf Stream) in equilibrium with such a density structure must have a velocity that increases with height. A system with inclined density surfaces has more potential energy than a system with horizontal density surfaces, just as a system with an inclined free surface has more potential energy that a system with a horizontal free surface. It is therefore potentially unstable, for it can release the stored potential energy by means of an instability that would cause the density surfaces to flatten out. In this case, vertical shear of the mean flow would decrease, and perturbations would gain kinetic energy (see also [16], [17], [18]).

Tests of atmospheric general-circulation models (GCMs) and, in particular, tests of their dynamical cores are important steps towards future model improvements. They reveal the influence of an individual model design on climate and weather simulations and indicate whether the circulation is described representatively by the numerical approach.

Thus stability of atmospheric jets (e.g. Gulf Steam in the Atlantic or Jet Streams) can be studied by means of kinetic and potential energy of the linearized mathematical models. However, it is an always 
open question what other quantities, rather than energy, can be conserved for nonlinear models. In this paper, we shall we shall obtain the symmetries associated with the nonlinear modeling describing spontaneous relaxation of eastward jets into a meandering state and use these symmetries for constructing the conservation laws by the method of nonlinear self-adjointness [13]. Similar analysis for the derivation of the conservation laws for the model of nonlinear atmospheric flows around the rotating spherical surface can be found in [14].

\section{Mathematical Model}

Instability of baroclinic jets that release potential energy by flattering out the density surfaces is called the baroclinic instability (see e.g. [19]). Two "baro-" words occur commonly in meteorology. A "barotropic" atmosphere is one in which the density depends only on the pressure; these are typically in the tropics. (Note the word "tropic" in this term.) A typical barotropic region is the southeast U.S. in the summer, or the tropics, where everyday is about the same: hot and humid. There are no weather fronts in a barotropic atmosphere. A "baroclinic atmosphere" is one in which the density of the atmosphere depends on both the temperature and the pressure; these are typically in the midlatitudes. Baroclinic atmospheres have distinct air masses of different temperatures with boundaries (frontal boundaries) between the two. There are density gradients at any level of the atmosphere in baroclinic environments.

We consider a basic state in which the density is stably stratified in the vertical with a uniform buoyancy frequency $N$, and increases northward at a constant rate $\partial \bar{\rho} / \partial y$. According to the thermal wind relation, the constancy of $\partial \bar{\rho} / \partial y$ requires that the vertical shear of the basic eastward flow $U(z)$ also be constant.

Let $\boldsymbol{u}=(u(x, y, z, t), v=v(x, y, z, t), w=w(x, y, z, t))$ be the velocity vector, $p(x, y, z, t)$ be the pressure and $\rho(x, y, z, t)$ be the density in the Cartesian coordinates $(x, y, z)$. The starting model describing the large-scale geophysical motions for baroclinic jets that release potential energy in the atmosphere are given by nonlinear model for internal waves under the Boussinesq approximation ([20], [21], [22], [23]):

$$
\begin{aligned}
\frac{\partial u}{\partial t}+u \frac{\partial u}{\partial x}+v \frac{\partial u}{\partial y}-f v & =-\frac{1}{\rho_{0}} \frac{\partial p}{\partial x}, \\
\frac{\partial v}{\partial t}+u \frac{\partial v}{\partial x}+v \frac{\partial v}{\partial y}+f u & =-\frac{1}{\rho_{0}} \frac{\partial p}{\partial y}, \\
\frac{\partial p}{\partial z}+\rho g & =0, \\
\frac{\partial \rho}{\partial t}+u \frac{\partial \rho}{\partial x}+v \frac{\partial \rho}{\partial y}+w \frac{\partial \rho}{\partial z} & =0, \\
\frac{\partial u}{\partial x}+\frac{\partial v}{\partial y}+\frac{\partial w}{\partial z} & =0,
\end{aligned}
$$

where $g$ is the acceleration due to gravity so that $p$ and $\rho$ are to be interpreted as the pressure and density departures from their mean state

$$
\bar{\rho}(y, z)=-\frac{\rho_{0}}{g} N^{2} z, \quad \bar{p}(y, z)=p_{0}-\rho_{0} g z-g \int_{0}^{z} \bar{\rho}(\xi) d \xi
$$

in which $\rho_{0}$ is the constant reference density, $\bar{\rho}(z)$ is a background stable density profile with the associated buoyancy frequency $N$ defined by

$$
N^{2}=-\frac{g}{\rho_{0}} \frac{d \bar{\rho}}{d z}
$$

and we require $\rho_{0}+\bar{\rho}$ and $\bar{p}$ to be consistent with the state of rest, i.e.,

$$
\frac{d \bar{p}}{d z}=-\left(\rho_{0}+\bar{\rho}\right) g
$$


The quantity $N$ measures the degree of density stratification of a fluid with average potential density $\rho(z)$ and thus represents the frequency with which a vertically displaced fluid element would be expected to oscillate because of restoring buoyancy forces. If the displacement is not strictly vertical, as in the case of internal waves, the restoring force is less, so the frequency of oscillations is reduced. The traditional $f$-plane approximation is made whereby we take $2 \vec{\Omega}=(0,0, f)$, where $f$ is the inertial frequency which depends on the rotation rate of the earth (angular velocity $\Omega=2 \pi \mathrm{rad} /$ day $\approx 0.73 \times 10^{-4} \mathrm{~s}^{-1}$ ).

We assume that the total flow is composed of the basic eastward jet $U(z)$ in geostrophic equilibrium with the basic density structure $\bar{\rho}(y, z)$, plus perturbations. That is

$$
\begin{aligned}
& u=U(z)+u^{\prime}(x, y, z, t), \quad v=v^{\prime}(x, y, z, t), \quad w=w^{\prime}(x, y, z, t), \\
& \rho=\bar{\rho}(y, z)+\rho^{\prime}(x, y, z, t), \quad p=\bar{p}(y, z)+p^{\prime}(x, y, z, t) .
\end{aligned}
$$

The basic flow is in geostrophic and hydrostatic balance:

$$
f U=-\frac{1}{\rho_{0}} \frac{\partial \bar{p}}{\partial y}, \quad 0=-\frac{\partial \bar{p}}{\partial z}-\bar{\rho} g .
$$

Eliminating the pressure, we get the thermal wind relation

$$
\frac{d U}{d z}=\frac{g}{f \rho_{0}} \frac{\partial \bar{\rho}}{\partial y}
$$

which says that the eastward flow must increase with height since $\partial \bar{\rho} / \partial y>0$.

In this paper, we find the symmetries and the associated conservation laws for the simplified model obtained by assuming that $\partial \bar{\rho} / \partial y$ is constant, so that the background flow is $U=U_{0} z$ with $U_{0}=$ const. As a result, we have the model described by the following system:

$$
\begin{aligned}
\frac{\partial u}{\partial t}+U_{0} z \frac{\partial u}{\partial x}-f v+\frac{1}{\rho_{0}} \frac{\partial p}{\partial x} & =-\left(u \frac{\partial u}{\partial x}+v \frac{\partial u}{\partial y}\right), \\
\frac{\partial v}{\partial t}+U_{0} z \frac{\partial v}{\partial x}+f u+\frac{1}{\rho_{0}} \frac{\partial p}{\partial y} & =-\left(u \frac{\partial v}{\partial x}+v \frac{\partial v}{\partial y}\right), \\
\frac{\partial p}{\partial z}+\rho g & =0 \\
\frac{\partial \rho}{\partial t}+U_{0} z \frac{\partial \rho}{\partial x}+\frac{f \rho_{0}}{g} U_{0} v-\frac{\rho_{0}}{g} N^{2} w & =-\left(u \frac{\partial \rho}{\partial x}+v \frac{\partial \rho}{\partial y}+w \frac{\partial \rho}{\partial z}\right), \\
\frac{\partial u}{\partial x}+\frac{\partial v}{\partial y}+\frac{\partial w}{\partial z} & =0 .
\end{aligned}
$$

Here $U_{0}, f, \rho_{0}$ and $N$ are constants. The system (2.1) has four independent variables $t, x, y, z$, and five dependent variables $u, v, w, p, \rho$.

\section{Nonlinear self-adjointness}

Recall the definition of the nonlinear self-adjointness [13]. Let

$$
F_{\alpha}\left(x, u, u_{(1)}\right)=0, \quad \alpha=1, \ldots, m,
$$

be a system of first-order partial differential equations with $n$ independent variables $x=\left(x^{1}, \ldots, x^{n}\right)$ and $m$ dependent variables $u=\left(u^{1}, \ldots, u^{m}\right)$. Here $u_{(1)}=\left\{u_{i}^{\alpha}\right\}$ denotes the set of the first-order partial derivatives:

$$
u_{i}^{\alpha}=\frac{\partial u^{\alpha}}{\partial x^{i}}, \quad \alpha=1, \ldots, m ; i=1, \ldots, n .
$$


The adjoint system to the system (3.1) has the form

$$
F_{\alpha}^{*}\left(x, u, v, u_{(1)}, r_{(1)}\right)=0, \quad \alpha=1, \ldots, m,
$$

where the adjoint operator $F_{\alpha}^{*}$ is defined by

$$
F_{\alpha}^{*}\left(x, u, r, u_{(1)}, r_{(1)}\right)=\sum_{i=1}^{n} D_{i}\left(\frac{\partial \mathcal{L}}{\partial u_{i}^{\alpha}}\right)-\frac{\partial \mathcal{L}}{\partial u^{\alpha}} .
$$

Here $r=\left(r^{1}, \ldots, r^{m}\right)$ is the set of new dependent variables, $\mathcal{L}$ is the formal Lagrangian given by

$$
\mathcal{L}=\sum_{\alpha=1}^{m} r^{\alpha} F_{\alpha}\left(x, u, u_{(1)}\right)
$$

and $D_{i}$ denotes the total differentiation:

$$
D_{i}=\frac{\partial}{\partial x^{i}}+u_{i}^{\alpha} \frac{\partial}{\partial u^{\alpha}}+u_{i j}^{\alpha} \frac{\partial}{\partial u_{j}^{\alpha}}+r_{i}^{\alpha} \frac{\partial}{\partial r^{\alpha}}, \quad i=1, \ldots, n .
$$

The system (3.1) is said to be nonlinearly self-adjoint if there exist a substitution

$$
r^{\alpha}=\varphi^{\alpha}(x, u), \quad \alpha=1, \ldots, m
$$

where not all $\varphi^{\alpha}$ vanish identically, such that the adjoint system (3.2) is satisfied for all solutions of the system (3.1) after eliminating the variables $r^{\alpha}$ by the substitution (3.4). This definition is equivalent to the requirement that the equations

$$
F_{\alpha}^{*}\left(x, u, \varphi, u_{(1)}, \varphi_{(1)}\right)=\lambda_{\alpha}^{\beta}(x, u) F_{\beta}\left(x, u, u_{(1)}\right)
$$

hold identically in $x, u, u_{(1)}$ for all $\alpha=1, \ldots, m$. Here $\lambda_{\alpha}^{\beta}(x, u)$ are undeterminate coefficients, $\varphi$ is the $m$-dimensional vector $\varphi=\left(\varphi^{1}(x, u), \ldots, \varphi^{m}(x, u)\right)$ and $\varphi_{(1)}=\left\{D_{i}(\varphi)\right\}_{i=1}^{n}$. The summation is assumed in $\beta=1, \ldots, m$.

We write the system (2.1) in the form (3.1),

$$
F_{\alpha}\left(x, u, u_{(1)}\right)=0, \quad \alpha=1, \ldots, 5
$$

with $x=(t, x, y, z), u=(u, v, w, p, \rho)$ and

$$
\begin{aligned}
& F_{1}=u_{t}+U_{0} z u_{x}-f v+\frac{1}{\rho_{0}} p_{x}+\left(u u_{x}+v u_{y}\right), \\
& F_{2}=v_{t}+U_{0} z v_{x}+f u+\frac{1}{\rho_{0}} p_{y}+\left(u v_{x}+v v_{y}\right), \\
& F_{3}=p_{z}+g \rho, \\
& F_{4}=\rho_{t}+U_{0} z \rho_{x}+\frac{\rho_{0}}{g}\left(U_{0} f v-N^{2} w\right)+\left(u \rho_{x}+v \rho_{y}+w \rho_{z}\right), \\
& F_{5}=u_{x}+v_{y}+w_{z},
\end{aligned}
$$

where the differentiations are denoted by the corresponding subscripts. Then we use the notation

$$
\begin{array}{ll}
u=u^{1}, & v=u^{2}, \quad w=u^{3}, \quad p=u^{4}, \quad \rho=u^{5}, \\
t=x^{1}, & x=x^{2}, \quad y=x^{3}, \quad z=x^{4}
\end{array}
$$


and obtain the following adjoint operators Eq. (3.3):

$$
\begin{aligned}
& F_{1}^{*}=r_{t}^{1}+\left(u+U_{0} z\right) r_{x}^{1}+v r_{y}^{1}+r_{x}^{5}-\left(f+v_{x}\right) r^{2}-\rho_{x} r^{4}+v_{y} r^{1}, \\
& F_{2}^{*}=r_{t}^{2}+\left(u+u_{0} z\right) r_{x}^{2}+v r_{y}^{2}+r_{y}^{5}+\left(f-u_{y}\right) r^{1}-\left(\rho_{y}+\frac{\rho_{0}}{g} f U_{0}\right) r^{4}+u_{x} r^{2}, \\
& F_{3}^{*}=\left(\frac{\rho_{0}}{g} N^{2}-\rho_{z}\right) r^{4}+r_{z}^{5}, \\
& F_{4}^{*}=\frac{1}{\rho_{0}}\left(r_{x}^{1}+r_{y}^{2}\right)+r_{z}^{3}, \\
& F_{5}^{*}=r_{t}^{4}+\left(u+u_{0} z\right) r_{x}^{4}+v r_{y}^{4}+w r_{z}^{4}+\left(u_{x}+v_{y}+w_{z}\right) r^{4}-g r^{3} .
\end{aligned}
$$

Solving the equations (3.5) with $F_{\alpha}$ and $F_{\alpha}^{*}$ given by the equations (3.7) and (3.9), respectively, we obtain the following substitution (3.4):

$$
\begin{aligned}
& r^{1}=0, \quad r^{2}=0, \\
& r^{3}=H_{1}^{\prime}, \quad r^{4}=g H_{2}^{\prime}+g H_{1}, \\
& r^{5}=H_{2}+\left(g \rho-N^{2} \rho_{0} z+f U_{0} \rho_{0} y\right) H_{1}+H_{3} .
\end{aligned}
$$

Here $H_{1}, H_{2}, H_{4}$ are arbitrary functions of one variable each,

$$
H_{1}=H_{1}(t), \quad H_{2}=H_{2}\left(g \rho-N^{2} \rho_{0} z+f U_{0} \rho_{0} y\right), \quad H_{3}=H_{3}(t) .
$$

The prime denotes the differentiation.

Thus, the system (3.6) is nonlinearly self-adjoint. The nonlinear self-adjointness condition (3.5) is satisfied in the following form:

$$
\begin{aligned}
& F_{1}^{*}=F_{2}^{*}=F_{3}^{*}=F_{4}^{*}=0, \\
& \left.F_{2}^{*}\right|_{r^{i}=\varphi^{i}}=0, \\
& F_{5}^{*}=H_{2} F_{4}+g\left(H_{2}^{\prime}-H_{1}\right) F_{5} .
\end{aligned}
$$

Now one can use the simple method for constructing conservation laws associated with symmetries of all nonlinearly self-adjoint equations [13]. According to this method, any infinitesimal symmetry

$$
X=\xi^{i}(x, u) \frac{\partial}{\partial x^{i}}+\eta^{\alpha}(x, u) \frac{\partial}{\partial u^{\alpha}}
$$

of the system (2.1) provides a conserved vector given by the formula

$$
C^{i}=\left(\eta^{\alpha}-\xi^{j} u_{j}^{\alpha}\right) \frac{\partial \mathcal{L}}{\partial u_{i}^{\alpha}}
$$

where $\mathcal{L}$ is the formal Lagrangian $\mathcal{L}=\sum_{\alpha=1}^{5} r^{\alpha} F_{\alpha}\left(x, u, u_{(1)}\right)$ of Eqs. (3.6). The auxiliary dependent variables $r^{\alpha}$ must be eliminated from the expressions (3.12) by means of the substitution (3.10).

\section{Symmetries}

The calculation shows that the symmetries of the system (2.1) is an infinite-dimensional Lie algebra with the basis 


$$
\begin{aligned}
X_{1} & =g z \frac{\partial}{\partial p}-\frac{\partial}{\partial \rho}, \quad X_{2}=\frac{\partial}{\partial z}-U_{0} \frac{\partial}{\partial u}+f \rho_{0} U_{0} y \frac{\partial}{\partial p} \\
X_{3} & =y \frac{\partial}{\partial x}-x \frac{\partial}{\partial y}+v \frac{\partial}{\partial u}-\left(U_{0} z+u\right) \frac{\partial}{\partial v}-f \rho_{0} U_{0} x z \frac{\partial}{\partial p}+\frac{\rho_{0}}{g} f U_{0} x \frac{\partial}{\partial \rho} \\
X_{4} & =z \frac{\partial}{\partial z}-U_{0} z \frac{\partial}{\partial u}+w \frac{\partial}{\partial w}+\rho_{0} z\left(U_{0} f y-N^{2} z\right) \frac{\partial}{\partial p} \\
& +\left[\frac{\rho_{0}}{g}\left(2 N^{2} z-f U_{0} y\right)-\rho\right] \frac{\partial}{\partial \rho}, \\
X_{5} & =x \frac{\partial}{\partial x}+y \frac{\partial}{\partial y}+z \frac{\partial}{\partial z}+u \frac{\partial}{\partial u}+v \frac{\partial}{\partial v}+w \frac{\partial}{\partial w}+2 p \frac{\partial}{\partial p}+\rho \frac{\partial}{\partial \rho} \\
X_{6} & =4 t \frac{\partial}{\partial t}+2(2 x+f t y) \frac{\partial}{\partial x}+(4 y-2 f t x) \frac{\partial}{\partial y}+2 f(t v+y) \frac{\partial}{\partial u} \\
& -2 f\left(U_{0} t z+x+t u\right) \frac{\partial}{\partial v}-4 w \frac{\partial}{\partial w} \\
& -f \rho_{0}\left[f x^{2}+f y^{2}+2 U_{0} z(f t x-2 y)\right] \frac{\partial}{\partial p}+2 \frac{\rho_{0}}{g} f U_{0}(f t x-2 y) \frac{\partial}{\partial \rho}, \\
X_{7} & =\frac{\partial}{\partial t}, \quad X_{8}=h_{1} \frac{\partial}{\partial x}+h_{1}^{\prime} \frac{\partial}{\partial u}-\rho_{0}\left(f y h_{1}^{\prime}+x h_{1}^{\prime \prime}\right) \frac{\partial}{\partial p}, \\
X_{9} & =h_{2} \frac{\partial}{\partial y}+h_{2}^{\prime} \frac{\partial}{\partial v}+\rho_{0}\left(U_{0} f z h_{2}+f x h_{2}^{\prime}-y h_{2}^{\prime \prime}\right) \frac{\partial}{\partial p}-\frac{\rho_{0}}{g} U_{0} f h_{2} \frac{\partial}{\partial \rho}, \\
X_{10} & =h_{3} \frac{\partial}{\partial p},
\end{aligned}
$$

where $h_{1}=h_{1}(t), h_{2}=h_{2}(t), h_{3}=h_{3}(t)$ are arbitrary functions.

\section{Conservation laws}

The conservation laws for Eqs. (3.6) will be written in the form

$$
D_{t}\left(C^{t}\right)+D_{x}\left(C^{x}\right)+D_{y}\left(C^{y}\right)+D_{z}\left(C^{z}\right)=\sum_{\alpha=1}^{5} \mu^{\alpha} F_{\alpha}
$$

where $\mu^{\alpha}$ are undetermined variable coefficients.

The components $C^{t}, \ldots, C^{z}$ of the conservation law (5.1) can be readily obtained by applying the conservation formulae (3.12) to the symmetries (4.1). The calculation shows that it is sufficient to use the symmetries $X_{1}, X_{5}$ and $X_{7}$. Combining the conserved vectors provided by $X_{1}, X_{5}, X_{7}$ and eliminating the trivial terms by the simplification procedure described in [13], we obtain the following conserved vector:

$$
\begin{aligned}
& C^{t}=g \rho H_{1}+H_{2}, \\
& C^{x}=g\left(U_{0} z+u\right) \rho H_{1}+\rho_{0}\left(f U_{0} y-N^{2} z\right) u H_{1}+U_{0} z H_{2}+u\left(H_{2}+H_{3}\right), \\
& C^{y}=\left(g \rho-N^{2} \rho_{0} z+f U_{0} \rho_{0} y\right) v H_{1}+v\left(H_{2}+H_{3}\right), \\
& C^{z}=p H_{1}^{\prime}+\left(g \rho-N^{2} \rho_{0} z+f U_{0} \rho_{0} y\right) w H_{1}+w\left(H_{2}+H_{3}\right),
\end{aligned}
$$

where the functions $H_{1}, H_{2}, H_{3}$ are given by Eqs. (3.11),

$$
H_{1}=H_{1}(t), \quad H_{2}=H_{2}\left(g \rho-N^{2} \rho_{0} z+f U_{0} \rho_{0} y\right), \quad H_{3}=H_{3}(t) .
$$

The remaining symmetries from (4.1) do not lead to new conserved vectors. Moreover, one can verify by the direct method, i.e. calculating conserved vectors from the definition (5.1) of the conservation 
law, that (5.2) is the most general form of the conserved vector whose components do not depend on derivatives of the dependent variables.

The vector (5.2) satisfies the conservation equation (5.1) with the coefficients $\mu^{\alpha}$ given by Eqs. (3.10),

$$
\begin{aligned}
& \mu^{1}=0, \quad \mu^{2}=0, \\
& \mu^{3}=H_{1}^{\prime}, \quad \mu^{4}=g H_{2}^{\prime}+g H_{1}, \\
& \mu^{5}=H_{2}+\left(g \rho-N^{2} \rho_{0} z+f U_{0} \rho_{0} y\right) H_{1}+H_{3} .
\end{aligned}
$$

Acknowledgements. The work of RNI on this research was supported in part by an appointment to the U.S. Department of Energy's Visiting Faculty Program at Pacific Northwest national Laboratory. NHI and LRG acknowledge the financial support of the Government of Russian Federation through Resolution No. 220, Agreement No. 11.G34.31.0042.

\section{References}

[1] R.F. Anderson, S. Ali, L.L. Brandtmiller, S.H.H. Nielsen, M.Q. Fleisher. Wind-driven upwelling in the Southern Ocean and the deglacial rise in atmospheric $\mathrm{CO}_{2}$. Science, 323 (2006), 1443-1448.

[2] R.D. Smith, M.E. Maltrud, F.O. Bryan, M.W. Hecht. Numerical Simulation of the North Atlantic Ocean at 1/10 . J. Phys. Oceanogr., 30 (2000), 1532-1561.

[3] R.N. Ibragimov. Nonlinear viscous fluid patterns in a thin rotating spherical domain and applications. Phys. Fluids, 23 (2010), 123102.

[4] R.N.Ibragimov, N.H. Ibragimov. Rotationally symmetric internal gravity waves. Int. J. Nonlinear Mech. 47, (2012) 46-52.

[5] R.N. Ibragimov. Generation of internal tides by an oscillating background flow along a corrugated slope. Phys. Scripta, $78,065801$.

[6] M.E. Maltrud, R. D. Smith, A. J. Semtner, R. C. Malone. Global Eddy-Resolving Ocean Simulations Driven by 19851994 Atmospheric Winds. J.Geophys. Res., 102 (1998), 25203-25226.

[7] R.N. Ibragimov. Free boundary effects on stability of two phase planar fluid motion in annulus: Migration of the stable mode. Comm. Nonlinear Sci. Numer. Simulat. 15 (2010), no. 9, 2361-2374.

[8] J. Frederiksen, P. Webster.Alternative Theories of Atmospheric Teleconnections and Low-Frequency Fluctuations. Rev. Geopphys. 26 (1998), no. 3, 459-494.

[9] R.N. Ibragimov, D.E. Pelinovsky. Incompressible viscous flows in a thin spherical shell. J. Math. Fluid Mech., 11 (2009), 60-90.

[10] N.H. Ibragimov, R.N. Ibragimov. Integration by quadratures of the nonlinear Euler equations modeling atmospheric flows in a thin rotating spherical shell. Phys. Lett. A., 375 (2011), 3858-3865.

[11] R.N. Ibragimov, M. Dameron, C. Dannangoda. Sources and sinks of energy balance for nonlinear atmospheric motion perturbed by west-to-east winds progressing on a surface of rotating spherical shell. Discontinuity, Nonlinearity and Complexity, 1 (2012), no. 1, 41-55.

[12] I. Held. 1978: The vertical scale of an unstable baroclinic wave and its importance for eddy heat flux parametrisations. J. Atmos. Sci., 35 (1978), 572-576.

[13] N.H. Ibragimov. Nonlinear self-adjointness in constructing conservation laws. Archives of ALGA 7/8, 1-99. See also arXiv:1109.1728v1[math-ph], 2011, 1-104.

[14] A.M. Araslanov, L.R. Galiakberova, N.H. Ibragimov, R.H. Ibragimov. Conserved vectors for a model of nonlinear atmospheric flows around the rotating spherical surface. Math. Model. Natur. Phenom., 8 (2013), no. 1, 32-48.

[15] W.K. Dewar. On "too fast" baroclinic planetary waves in the general circulation. J. Phys. Oceanogr., 29 (1998), 500-511.

[16] R.N. Ibragimov, G. Jefferson, J. Carminati. Explicit invariant solutions associated with nonlinear atmospheric flows in a thin rotating spherical shell with and without west-to-east jets perturbations. Spinger: Anal. Math. Phys., 3 (2013), no. 3, 201-294.

[17] N.H. Ibragimov, R.N. Ibragimov. Nonlinear whirlpools versus harmonic waves in a rotating column of stratified fluid. Math. Model. Nat. Phenom., 8 (2013), no. 1, 32-14.

[18] R.N. Ibragimov, M. Dameron. Spinning phenomena and energetics of spherically pulsating patterns in stratified fluids. Phys. Scripta 84, 2011015402.

[19] A.J. Simmons, B.J. Hoskins. Baroclinic instability on the sphere: Normal modes of the primitive and quasi-geostrophic equations. J. Atmos. Sci., 33 (1975), 1454-1477.

[20] J. Pedlosky. Geophysical Fluid Dynamics. Springer-Verlag, Second Edition, (1986).

[21] N.H. Ibragimov, R.N. Ibragimov. Applications of Lie Group analysis to mathematical modeling in natural sciences". Math. Model. Nat. Phenom., 7 (2012), no. 2, 52-65.

[22] R.N. Ibragimov. Mechanism of energy transfers to smaller scales within the rotational internal wave field. Springer: Math. Phys. Anal. Geom., 13 (2010), no. 4, 331- 355.

[23] R.N. Ibragimov. Shallow water theory and solution of the problem on the atmospheric motion around a celestial body. Phys. Scripta., 61 (2000), 391-395. 